\title{
Reflexiones de la Investigación en La Formación de Maestros De Biología: Una Propuesta desde la Pedagogía.
}

\section{Meditations of the Investigation in The Biology Teacher's Formation: A Proposal from the Pedagogy.}

\section{Por: Sanchez Reyes Leslie Alexa ${ }^{1}$}

\begin{tabular}{|l|}
\hline Recibido: 25- 09-2009 \\
\hline Aceptado: 26-11-2009 \\
\hline
\end{tabular}

"No hay enseñanza sin investigación ni investigación sin enseñanza. Esos quehaceres se encuentran cada uno en el cuerpo del otro. Mientras enseño continúo buscando, indagando, enseño porque busco, porque indagué, porque indago y me indago..."

(Freire, 1997)

\section{Resumen}

El presente escrito pretende abordar la enseñanza de la biología desde la investigación en la formación de maestros de este campo, es así como la investigación, tan indispensable en la práctica del maestro, permite la configuración de otras miradas para la construcción de saber pedagógico y biológico que lleva consigo las reflexiones y por lo tanto el reconocimiento de un contexto, razón por la cual vale la pena revisar el lugar de la investigación en el contexto escolar colombiano haciendo un llamado a la reconceptualización de "la pedagogía como saber histórico construido por el maestro" (Zuluaga, 1999).

Palabras clave: Investigación, Maestro, Pedagogía, Práctica pedagógica, Saber pedagógico y biológico, Gestión, Contexto escolar.

\begin{abstract}
This writing pretend to approach the biology's teaching from the investigation in the teacher's formation of this field, it is as well as the investigation, so indispensable in the teacher's practice, let the configuration of the other glances for the construction of pedagogic and biologic knowledge, that include reflections and consequently the recognition of a context, for this reason worthwhile revise the position of the investigation in the Colombian school context calling to the conceptualization of "the pedagogy like historic knowledge built by the teacher" (Zuluaga, 1999)
\end{abstract}

Keywords: Investigation, Teacher, Pedagogy, Pedagogy's practice, Pedagogic and Biologic Knowledge, Step, School Context.

${ }^{1}$ Estudiante de Licenciatura en Biología. Universidad Pedagógica Nacional.farukalexa@hotmail.com 
Desde el espacio que el Proyecto Curricular de Licenciatura en Biología otorga para los seminarios de Métodos de Investigación en Quinto Semestre, surgieron reflexiones alrededor de la investigación en la formación de maestros de biología, es así como este ensayo pretende pensar la investigación desde un lugar que vale la pena reconceptualizar porque se constituye en el saber propio del maestro: La pedagogía.

La formación investigativa en los maestros tiene la urgencia de posibilitar reflexiones alrededor de su práctica y su constitución como sujeto ético y político con un compromiso social, es por esto que tanto la construcción de saber pedagógico como de los campos disciplinares específicos, para el caso de la biología, se constituye en posibilidad para reconocer otras miradas enmarcadas en la realidad y que permiten pensarse y pensar al otro. En este sentido, vale la pena dedicar un espacio para reflexionar acerca de la investigación en la formación de maestros de biología, teniendo como punto de partida una propuesta desde la pedagogía, porque es ésta la que históricamente ha construido el maestro para pensar su ser y su quehacer.

Primero es importante aclarar que más allá de lo instrumental de lo que pareciera estar reducida, la apuesta por la pedagogía es un reto que asumen los que saben que su campo de acción lo constituye "la enseñanza como posibilidad de pensamiento" (Martínez, 2003) y punto de problematización para la reflexión de su propia práctica, de modo que desde esta posición es el maestro el sujeto capaz de pensar y reflexionar en una mirada de sí mismo, para así transformarse y construir otras miradas sobre una realidad existente y a partir del reconocimiento de otros participar activamente en la formación de sujetos, esa posibilidad de pensamiento se constituye gracias a la investigación, y ésta pensada desde la pedagogía le otorga el carácter de indispensable en la reflexión pedagógica y, por supuesto, en práctica cotidiana del maestro.

Teniendo en cuenta la importancia de la formación investigativa en los maestros, es preciso reconocer en la investigación pedagógica los diferentes enfoques teóricos y epistemológicos que han tomado postura alrededor de los objetos y los sujetos de investigación así como las relaciones entre éstos para que el maestro pueda comprender las diferentes formas de investigación y los elementos que la configuran dependiendo de las condiciones que se presenten, de manera que pueda ubicar su reflexión dentro de un contexto y una realidad existente a partir de la construcción del objeto de investigación.

Al hablar del contexto, se hace pertinente ubicar la investigación en el escenario escolar colombiano y el lugar que tiene el maestro de biología como sujeto investigador en la escuela, es así como surgen problematizaciones desde la gestión entendida como una forma de gobierno de la práctica educativa que constituye en gran medida la práctica docente y que actualmente es validada en términos de producción y competencias, respondiendo a su vez a unas lógicas globales que dejan cuestionamientos alrededor de la función crítica, reflexiva y transformadora de la investigación; el sentido de la enseñanza; y el lugar del saber pedagógico.

Si bien es cierto que el saber es indispensable a la constitución de la pedagogía, entendiendo éste como "el espacio más amplio y abierto de un conocimiento, donde se pueden localizar discursos de muy diferentes niveles" (Foucault, 1970), vale la pena 
preguntar ¿Qué lugar tiene el saber pedagógico en la investigación pedagógica? Según Zuluaga (2003) Hoy en día, cubre no sólo las teorías sobre educación y la enseñanza (ciencias de la educación, pedagogía y didáctica), sino también la cotidianidad de la enseñanza, las acciones prácticas del maestro, la organización de la escuela, los contenidos que se imparten en las instituciones formadoras de docentes. Dentro de este contenido del saber pedagógico hay que reconocer que ofrece las bases para la problematización de la pedagogía y por supuesto su conceptualización a partir del fortalecimiento de su autonomía en el campo de la enseñanza, de modo que la formación en investigación pedagógica tiene la urgencia de llevar a cabo procesos de investigación en los maestros para revalidar su campo de acción una vez que se consolida el saber.

Ahora es preciso considerar la diferencia entre el saber y el conocimiento ya que a partir de allí se puede reconocer el lugar del maestro en la enseñanza y por supuesto en la investigación, puesto que el saber hace referencia a una dimensión más amplia que comprende a su vez, como ya lo había mencionado, discursos de diferentes niveles, y prácticas, implica además de la dimensión cognoscitiva, una dimensión axiológica, y surge de un proceso singular de experiencia en medio de la interacción social; mientras que el conocimiento es resultado de un proceso intelectual, que es validado por una comunidad intelectual de la disciplina específica, y por lo tanto hace parte de la dimensión cognitiva que comprende el saber, queda claro entonces que los elementos que parten del saber involucran la singularidad de los sujetos y en este sentido logra movilizar prácticas y discursos que van más allá del conocer, en este sentido, el maestro es quien puede posibilitar construcciones de saberes, que además, desde los procesos intelectuales generen también nuevas configuraciones de sujeto y miradas diferentes de la realidad.

Una vez manifiesta esta diferencia es importante ubicar el lugar de la enseñanza como propia del maestro, porque es su posibilidad de pensamiento, así como de los otros y de las relaciones con los otros, de ahí parte su reflexión e inflexión, y es en este sentido donde se reconoce aún más el trabajo investigativo en la construcción de nuevas miradas, configurando su saber pedagógico a través de su práctica, los sujetos que la constituyen y la reflexión de saber que implica desde la pedagogía.

Sin dejar de lado el campo disciplinar en el que se desempeña ese sujeto maestro, la biología se constituye como uno de los objetos a partir de los cuales problematiza su enseñanza, pero este "objeto" en particular se piensa desde su configuración como ciencia, y de esta manera en las diferentes formas de comprender los fenómenos de lo vivo; desde la enseñanza de la biología se pueden construir saberes que permitan hacer de lo vivo una múltiple configuración de miradas con sentido y de las cuales se apropian los sujetos mediante discursos y prácticas que salgan de los lugares comunes, este es el punto en común entre la construcción de saber pedagógico y biológico, y es desde allí que el sujeto se reconoce como productor, constructor de saberes que él mismo apropia y que no sólo reproduce.

Cuando la enseñanza de la biología toma esta postura surge la pregunta del maestro por el qué enseñar, y particularmente, qué enseñar en ciencias, tal vez la respuesta a estas preguntas tiene estrecha relación con ciertos contenidos correspondientes a la disciplina como tal, los que hacen referencia a los conocimientos que ya han sido validados, sin 
embargo no hay que perder de vista que alrededor de éstos se configuran formas singulares de pensamiento que dan cuenta de otros enfoques y que generan diferentes procesos de construcción de conocimiento y a su vez de saberes, que responden, más allá de las dinámicas de las competencias (saber hacer en contexto), a su propio proceso de formación; es así como en la enseñanza intervienen sujetos y saberes particulares que la hacen objeto de reflexión desde la cual parte su formación.

Es a partir de la formación en investigación que el maestro reconoce su lugar en la enseñanza, debido a su posibilidad de transformar y de ser dueño del conocimiento que él mismo produce para su propio saber pedagógico y biológico, que consolida a partir de su campo de acción en la práctica pedagógica, y que problematiza constantemente a partir de la reflexión pedagógica.

Para el caso de la formación investigativa del programa de formación en la Licenciatura de Biología, ésta tiene que ver con la apropiación de la investigación en sus múltiples formas de abordarla a partir de la construcción de proyectos de investigación, que generan a su vez procesos con unos niveles de elaboración en los ambientes de formación y los diferentes espacios académicos de cada eje curricular, proyectos pensados desde la problematización de los núcleos integradores de problema (NIPs); es por esto que vale la pena reconocer el nivel de investigación que se ha llevado a cabo con el desarrollo de los proyectos semestrales, lo cual tiene en cuenta, además de la producción de conocimientos a manera de informe, los métodos y procedimientos, el sustento teórico y problemático, los procesos que dan cuenta de los resultados y su correspondiente análisis, etc., y por último los saberes construidos desde su desarrollo para aplicarlo en las diferentes áreas de formación disciplinar, humanística, y pedagógica.

En este sentido, la investigación referente a lo riguroso y procedimental, adopta ciertos enfoques o métodos que tienen que ver con el cómo se construye el conocimiento, a partir de los paradigmas desde los cuales ha sido posible asumir las dimensiones que comprende el proceso de investigación, es decir, que el tipo de reflexividad que se construye depende en gran medida de la metodología y la orientación de las dinámicas que se llevan a cabo; no obstante, no hay que olvidar el lugar del investigador en estos procesos, "se asume al investigador como sujeto del conocimiento, ligado a experiencias y a proyectos; sujeto portador de una historia que configura su dinámica de relaciones; es desde estas dinámicas donde se generan los temas y problemas que impulsan el trabajo de la investigación dando orientación y sentido a su práctica social y profesional."(ITAE), entonces, para el investigador es importante asumir la lógica de lo metodológico siempre desde un proceso reflexivo, que más allá de los pasos procedimentales pueda reconocer y problematizar su propia construcción investigativa.

En la misma lógica, la formación investigativa, y más aún de los maestros de una ciencia como la biología, debería no sólo responder a la elaboración de proyectos en términos de productos, procedimientos operativos, o comprobaciones mecánicas de fenómenos ya estudiados, sino en las construcciones singulares generadas que comprometen otras miradas en la configuración de su ser, sus saberes y sus prácticas; por tanto, la investigación como un proceso formativo individual ofrece todos los elementos para 
construir conocimiento por sí mismos, nuevas formas de ver el mundo, la realidad y lo vivo, pero que puedan trascender a construcciones colectivas.

Como parte de la formación investigativa, es importante valorar que un proyecto de investigación se lleva a cabo a partir de problematizaciones e intereses, que surgen del reconocimiento de un contexto que configura a su vez una realidad, es así como el sujeto investigador se asume como agente social crítico, e incluso el maestro también se configura desde la investigación como actor principal en la formación de sujetos en su disciplina a enseñar, y en las demás dimensiones que implica la enseñanza como punto de problematización de su práctica pedagógica.

Ahora bien, al reconocer la investigación como práctica indispensable en la reflexión pedagógica, no hay que perder de vista que en el contexto escolar en Colombia la investigación educativa supone un sentido totalmente diferente, "la aparición y consolidación de la investigación educativa en Colombia fue uno de los múltiples mecanismos que generó la estrategia de desarrollo una vez inició su despliegue en la década de $1950 \ldots$ ha tenido una función política de primer orden... ha contribuido a la producción de la realidad educativa nacional" (Noguera, 2002) y a partir de esta realidad creada, denominada "subdesarrollo", se han establecido ciertos discursos y prácticas que son objeto de "estudios del desarrollo" por parte de "expertos" orientados a producir regímenes y puesta en marcha de políticas en torno a la excelencia, competitividad y acreditación que son de carácter internacional.

De acuerdo a estas condiciones, es importante resaltar la manifestación del Movimiento Pedagógico en contra de la tendencia economista que rige la educación y que ha reemplazado a la pedagogía por un ejercicio de "gestión" en el que el lugar del maestro se encuentra relegado a una "gerencia" que no le corresponde; es por esto que el movimiento pedagógico, además de ser reconocido como único en Latinoamérica, ha dejado sus mayores contribuciones en la reconceptualización del campo pedagógico en la medida que orientó la investigación, que surge de una realidad existente nacional, hacia la pregunta por la escuela, el maestro, la enseñanza, y la pedagogía.

A pesar de estar sujeto a una gestión escolar, el maestro como sujeto ético y político tiene la posibilidad de pensarse en su interior, y pensar en su afuera, de este modo, vale la pena preguntar ¿Qué debemos investigar los maestros en Colombia hoy?, y es que, en este sentido habría que resaltar aún más la función crítica, reflexiva y transformadora de la investigación, para no responder siempre a las lógicas de productividad y rentabilidad, sino permitir nuevos enfoques, miradas, perspectivas que desde su reconocimiento puedan comprometer ciertas prácticas y discursos en la configuración del ser y del quehacer del maestro.

Para precisar algunas conclusiones de este espacio de reflexiones, que si bien es cierto debería estar presente continuamente como práctica cotidiana, quedan por ahora nuevas inquietudes y problematizaciones, acerca de sí mismo, de la coherencia de los principales propósitos de la investigación con la administración y gestión de proyectos, del reconocimiento de los saberes como construcciones propias validadas por los sujetos, de la pedagogía como un saber histórico construido que vale la pena reconceptualizar desde la 
enseñanza, de la enseñanza que parece invisible bajo la lógica del aprendizaje, del maestro como intelectual pero sustituible, de la biología como posibilidad de configuración de mi visión de mundo en torno a lo vivo y mi lugar en él, de lo vivo como el objeto particular de la ciencia que al igual que los sujetos no se reduce a leyes universales, etc.

La apuesta por la pedagogía supone una configuración de maestro, enseñanza, objeto de enseñanza, y saber pedagógico, a partir de un proceso reflexivo continuo y movilizador, en el que se puedan constituir nuevas miradas que trasciendan de los lugares comunes y que le permitan a los sujetos construir saberes por sí mismos, ese es el compromiso que tenemos como maestros, y es la investigación permanente la que lo posibilita, una investigación que nace de los sujetos y por supuesto de su realidad, y una investigación desde la pedagogía que se hace indispensable en la reflexión de mi ser y quehacer como maestra, y constructora de país.

\section{BIBLIOGRAFÍA}

- Freire, P. (1997). Pedagogía de la autonomía. Saberes necesarios para la práctica educativa. Madrid: Siglo XXI Editores.

- Foucault, M. (1970). La arqueología del saber. México: Siglo XXI Editores,.

- ITAE. “Aspectos Generales Módulo Investigación”. Instituto de tecnologías en Educación y a Distancia UPN. Elaborado por Equipo ITAE.

- Martínez, A. (2003). La enseñanza como posibilidad de pensamiento. En: Pedagogía y Epistemología. Grupo Historia de las Prácticas Pedagógicas. Bogotá: Ed. Magisterio.

- Zuluaga, O. (1999). Pedagogía e historia. La historicidad de la pedagogía. Antioquia: Antropos.

- Zuluaga, O. (2003). El florecimiento de las investigaciones pedagógicas. En Pedagogía y epistemología. Colombia: Colección Pedagogía e Historia. Grupo Historia de la Práctica Pedagógica. 